\title{
CRISIS, EMPLEO Y DESEQUILIBRIOS URBANOS EN ESPAÑA ${ }^{1}$
}

\section{CRISIS, EMPLOYMENT AND URBAN IMBALANCE IN SPAIN}

\author{
Gema González- Romero \\ Universidad de Sevilla \\ gemagonzalez@us.es \\ Inmaculada Caravaca Barroso \\ Universidad de Sevilla \\ caravaca@us.es \\ Paloma López-Lara \\ Instituto de Estadística y Cartografía de Andalucía \\ lopezlara@gmail.com
}

Recibido: octubre, 2014.

Versión final aceptada: diciembre, 2014.

PALABRAS CLAVE: Crisis, empleo, ciudad, España.

KEY WORDS: Crisis, employment, cities, Spain.

RESUMEN

Entre los múltiples impactos que la crisis económica está provocando destacan los que afectan al empleo. Resulta de especial interés lo que está ocurriendo en los espacios urbanos, al ser en ellos donde se concentran las actividades económicas y la población. En este contexto, el objetivo de este artículo es analizar la evolución experimentada por el empleo en las ciudades españolas con más de 20.000 habitantes, diferenciando entre dos fases: la comprendida entre los años 2006 y 2009, en la que se inicia la crisis, y la que trascurre entre 2009 y 2012, cuando empiezan a detectarse los efectos de las políticas de austeridad, que están contribuyendo a generar un fuerte crecimiento de las desigualdades.

\section{ABSTRACT}

Among the many impacts of the economic crisis is reducing stresses experienced by employment. This evolution can be seen more clearly in urban areas, where they highlight the uneven spatial effects of the crisis.

In this context, the aim of this paper is to analyze the evolution of employment in the Spanish towns and cities with over 20,000 inhabitants, differentiating between two phases: between 2006 and 2009, where the crisis began, and which elapses between 2009 and 2012, when the effects of the austerity policies that are contributing to a strong growth of inequality begin to be detected.

1 Este artículo se integra en el proyecto de investigación del Plan Nacional de I+D+i: CSO 2012-36170.

ISSN: 0212-8594 ISSN-e: 2340-2776.№ DOI: http://dx.doi.org/10.12795/rea.2015.i32.06

REA 32 (2015):134-150

http://editorial.us.es/es/revista-de-estudios-andaluces 


\section{INTRODUCCIÓN.}

Entre los múltiples efectos adversos que la crisis está provocando destaca con mucho la fuerte reducción experimentada por el número de empleos en buena parte de los países desarrollados y muy especialmente en algunos de los que conforman la Unión Europea. Como es sabido, este hecho está directamente relacionado por una parte, con el proceso de reestructuración del desregulado e hipertrofiado sector financiero y, por otra, con el estallido de la llamada burbuja inmobiliaria, procesos que vienen siendo profusamente analizados desde diferentes disciplinas y perspectivas (Etxezarreta, M. 2009; Corpataux, J., Crevoisier, O. y Theurillat, T. 2009; Fernández Durán, R. 2011; Harvey, D. 2012a; Daher, A. 2013).

Pero, junto a las causas de base antes citadas, las fuertes pérdidas de empleos deben asociarse también a las políticas neoliberales que la Unión Europea está aplicando como respuesta a la crisis. En efecto, las medidas de austeridad que, basadas en la desregulación del mercado laboral y en la privatización de servicios públicos, están siendo impuestas por las instituciones comunitarias a los países más castigados por la crisis, tienen un claro matiz ideológico y no sólo no están contribuyendo a resolver los graves problemas que en ellos se han venido generando, sino que han dado lugar, además, a la conformación de una segunda crisis (Dévoluy, $M$ et al, 2012; Lordon, $F$. , 2012; Álvarez Peralta, I, Luengo Escalonilla, F. Uxó González, J., 2013). Como se ha enfatizado al respecto, no deja de resultar paradójico que, mientras se utiliza el argumento de que estas políticas son imprescindibles para resolver la crisis, las medidas que se están implementando no se centren en controlar las inadecuadas prácticas financieras responsables de haber puesto en riesgo al sistema económico a escala global, sino que se limiten a gestionar sus efectos "eludiendo de forma evidente la posibilidad de combatir sus causas" (Lordon, F., 2012, 254).

Hay que tener en cuenta, además, que como consecuencia de dichas políticas, la cohesión socio-territorial de los países de la Unión Europea, y muy especialmente de los que conforman la Zona Euro, está experimentando un fuerte retroceso al castigar a los más vulnerables mientras benefician a los más competitivos, lo que lógicamente contribuye a provocar crecientes divergencias entre los Estados miembros. Constituye un buen ejemplo de lo anterior que "España, Portugal y Grecia han sido penalizados con una pérdida de producción de entre un 5 y un $10 \%$ del PIB, y Francia con una pérdida del $6 \%$, mientras Alemania, por el contrario, se beneficiaba de un efecto favorable equivalente al $8 \%$ de su PIB" (Dévoluy, M. et al, 2012, 56).

Se pone así en evidencia que la crisis esté incidiendo con distintas intensidades y formas en los territorios, contribuyendo, a su vez, a generar en ellos nuevos desequilibrios, desigualdades y contradicciones. No hay que olvidar que, como dice Harvey, D. (2012a), las diferencias locales ejercen un papel fundamental en la configuración del espacio del capital; es lo que Martin llama la glocalización de la crisis (Martin, R., 2011). 
Siendo esto así, resulta imprescindible considerar dicha escala tanto para profundizar en el conocimiento de las diferentes formas con que los territorios reaccionan ante la crisis, como para entender por qué ciertos ámbitos locales están siendo especialmente vulnerables a ella, mientras otros se ven menos afectados o muestran una mayor capacidad para hacerle frente.

Teniendo en cuenta lo anterior, los espacios urbanos parecen especialmente ilustrativos para analizar estos impactos. Si, por una parte, las ciudades concentran la población, las empresas, las instituciones, los conocimientos y las innovaciones que contribuyen a dinamizar la economía en periodos de crecimiento, por otra, son también dichos ámbitos los que resultan más afectados en periodos de recesión. No puede extrañar, por consiguiente, que se haya enfatizado acerca de las raíces urbanas de las crisis financieras (Harvey, D., 2012 b; Méndez, R., 2013 a y b).

En este contexto general de referencia, el objetivo de este artículo es analizar la evolución experimentada por el empleo en las ciudades españolas con más de 20.000 habitantes; no obstante, dada la disponibilidad de información, la unidad espacial de referencia utilizada es el municipio, por lo que indistintamente se hace alusión a ciudades y a municipios urbanos. La fuente básica utilizada es el Registro General de la Seguridad Social, que contiene información municipalizada que ha sido seleccionada para los años 2000, 2006, 2009 y 2012, lo que permite diferenciar entre lo ocurrido en los últimos años de la etapa económica expansiva previa a la crisis, y lo sucedido después durante otras dos fases muy significativas: la comprendida entre los años 2006 y 2009, en la que se inicia la crisis financiero-inmobiliaria, y la que trascurre entre 2009 y 2012 , cuando empiezan a detectarse los efectos de las llamadas políticas de austeridad.

No hay que olvidar que en España, junto a los efectos originados por la explosión de la burbuja financiero-inmobiliaria, las políticas aplicadas para enfrentar la crisis se están centrando en la reducción del déficit en la balanza de pagos a través de la minimización del gasto público, sin tener en cuenta que el problema de dicha balanza no está relacionado con los gastos sino con los ingresos, de forma que la recaudación fiscal favorece sistemáticamente a las grandes fortunas mientras las medidas de control al fraude no resultan operativas (Ayala, L., Martínez, R. y Ruiz Huerta, J., 2013; Romero, J., Collado, J. C., Rodríguez, M. T., 2014). De este modo, a la pérdida de empleos producida durante la primera fase de la crisis, estrechamente relacionada con la evolución experimentada por los sectores inmobiliario y financiero, se añade una segunda fase en la que los empleos se pierden fundamentalmente en servicios a la población, algunos tan básicos como la educación y la sanidad que en buena parte están asociados al sector público.

El artículo se estructura en siete epígrafes. Tras esta introducción, se hace referencia a la localización del empleo en los municipios urbanos españoles según tamaños. Los apartados tres, cuatro y cinco se dedican respectivamente a analizar las consecuencias territoriales de la evolución experimentada por los empleos (epígrafe tres), para 
considerar después los desequilibrios interurbanos generados primero por la crisis financiero-inmobiliaria y después por las políticas de ajuste (apartados cuatro y cinco). Por su parte, en el apartado seis se propone una tipología de las ciudades según los efectos provocados en ellas por la crisis. Por último se recogen algunas conclusiones.

\section{CRISIS Y CAMBIOS EXPERIMENTADOS POR LA DISTRIBUCIÓN DEL EMPLEO EN LOS MUNICIPIOS URBANOS SEGÚN SUS TAMAÑOS.}

España es uno de los países europeos cuyo comportamiento del empleo está siendo más preocupante en los últimos años; lo que se pone en evidencia al constatar que entre los años 2006 y 2012 el número de afiliados a la Seguridad Social se ha reducido en 2.437.772 efectivos (Registro General de la Seguridad Social). Este hecho se produce, además, en un país con una demostrada incapacidad estructural para generar el suficiente número de empleos (Toharia, L., 1992; Castaño, C., 1994; Etxezarreta, M., 1995; Méndez, R., 1998...), por lo que no resulta extraño que, lamentablemente, encabece el ranking del desempleo (López, I. y Rodríguez, E. 2010).

Según muestra el cuadro 1, se sigue manteniendo el tradicional desequilibrio económico entre los municipios que conforman el sistema urbano español, puesto que sólo en los que superan los 100.000 habitantes el peso relativo de las afiliaciones a la Seguridad Social supera al de la población. Por su parte, los cambios que se han ido produciendo a lo largo del tiempo muestran distintos grados de incidencia de la crisis según tamaño municipal. Durante los primeros años de la crisis (2006-2009), la reducción del número de empleos fue especialmente intensa tanto en los municipios urbanos de menor tamaño $(-9,55 \%)$, como en los mayores $(-6,59 \%)$. No obstante, se han ido produciendo ciertos cambios durante el siguiente periodo (2009-2012) en el que el descenso del número de afiliaciones se intensifica en los municipios entre 50.000 y 500.000 habitantes, mientras se frena tanto en los de menor tamaño como en los mayores. Respecto a la tasa de actividad, considerando en esta ocasión para medirla la relación entre las afiliaciones a la Seguridad Social y la población en edad activa², se comprueba cómo destacan los municipios mayores de 100.000 habitantes, que son los que alcanzan cifras superiores al $50 \% \mathrm{y}$, muy especialmente, los de mayor tamaño que llegan a alcanzar casi el $75 \%$.

\footnotetext{
${ }^{2}$ Al no disponer de información municipalizada sobre población activa para todos los años, se utiliza la potencialmente activa, es decir, aquella con una edad comprendida entre los 16 y los 65 años.
}

ISSN: 0212-8594 ISSN-e: 2340-2776.N№ DOI: http://dx.doi.org/10.12795/rea.2015.i32.06

REA 32 (2015):134-150

http://editorial.us.es/es/revista-de-estudios-andaluces 
Cuadro 1. Afiliaciones a la Seguridad Social en los municipios urbanos según tamaños.

\begin{tabular}{|l|c|c|c|c|c|}
\hline Tamaño municipal & $\begin{array}{c}\text { Población } \\
\mathbf{2 0 1 2}\end{array}$ & $\begin{array}{c}\text { Afiliaciones SS, } \\
\mathbf{2 0 0 6 - 2 0 0 9}\end{array}$ & $\begin{array}{c}\text { Afiliaciones SS, } \\
\mathbf{2 0 0 9 - 2 0 1 2}\end{array}$ & $\begin{array}{c}\text { Afiliaciones SS, } \\
\mathbf{2 0 1 2}\end{array}$ & $\begin{array}{c}\text { Afiliaciones } \\
\text { SS/Pob. Edad } \\
\text { Activa, 2012 }\end{array}$ \\
\hline $20.001-50.000$ & $\begin{array}{c}7.561 .192 \\
16 \%\end{array}$ & $-9,55 \%$ & $-7,63 \%$ & $\begin{array}{c}2.122 .947 \\
13,00 \%\end{array}$ & $41,42 \%$ \\
\hline $50.001-100.000$ & $\begin{array}{c}5.896 .687 \\
12,48 \%\end{array}$ & $-5,74 \%$ & $-6,87 \%$ & $\begin{array}{c}1.969 .235 \\
12,06 \%\end{array}$ & $49,02 \%$ \\
\hline \multirow{2}{*}{$100.001-250.000$} & $\begin{array}{c}7.803 .658 \\
16,51 \%\end{array}$ & $-5,78 \%$ & $-7,36 \%$ & $\begin{array}{c}2.828 .022 \\
17,32 \%\end{array}$ & $53,52 \%$ \\
\hline \multirow{2}{*}{$250.000-500.000$} & $\begin{array}{c}3.390 .092 \\
7,17 \%\end{array}$ & $-5,93 \%$ & $-7,66 \%$ & $\begin{array}{c}1.347 .552 \\
8,25 \%\end{array}$ & $59,22 \%$ \\
\hline$>500.000$ & $\begin{array}{c}7.600 .910 \\
16,08 \%\end{array}$ & $-6,59 \%$ & $-6,09 \%$ & $\begin{array}{c}3.762 .040 \\
23,03 \%\end{array}$ & $74,55 \%$ \\
\hline Total >20.000 & $\begin{array}{c}32.252 .539 \\
68,24 \%\end{array}$ & $-6,73 \%$ & $-6,97 \%$ & $\begin{array}{c}12.029 .796 \\
76,66 \%\end{array}$ & $55,31 \%$ \\
\hline Total España & $\mathbf{4 7 . 2 6 5 . 3 2 1}$ & & & $\mathbf{1 6 . 3 3 2 . 4 8 8}$ & $\mathbf{1 0 0 \%}$ \\
\hline
\end{tabular}

Fuente: Tesorería General de la Seguridad Social, Padrón Municipal de Habitantes, INE y elaboración propia.

\section{DISTRIBUCIÓN TERRITORIAL DE LOS CAMBIOS EXPERIMENTADOS POR EL EMPLEO.}

Para poder observar con más nitidez los impactos territoriales de la crisis asociados a las pérdidas de empleos producidas en las ciudades objeto de estudio, resulta de interés analizar los cambios que se han venido produciendo desde que se inicia el milenio. Hay que partir de la base de que se trata de transformaciones que suelen ser lentas y que, en consecuencia, no son fáciles de apreciar y mucho menos de caracterizar en cortos periodos de tiempo, no obstante, permiten ya apreciar ciertas tendencias.

En primer lugar, se ha considerado lo ocurrido entre los años 2000 y 2006, es decir en la fase económica expansiva que antecede a la crisis y que, como es bien sabido, estuvo sustentada en buena parte por un desmedido crecimiento del sector inmobiliario. Como puede observarse en la figura 1 , resulta bastante llamativo el crecimiento experimentado durante dichos años por el número de afiliaciones a la Seguridad Social en la práctica totalidad de las ciudades, pues, únicamente dos municipios: Colmenar Viejo en Madrid y Sestao en Vizcaya, experimentaron pérdidas. En el primero de los casos se trata de un error de la base de datos que en el año 2000 sigue incluyendo en Colmenar empleos que pertenecían a Tres Cantos, pese a que este último municipio se había segregado de él en 1991. En el caso de Sestao las pérdidas se explican por los procesos de reestructuración y privatización de empresas públicas industriales que durante esos años se estaban llevando a cabo.

ISSN: 0212-8594 ISSN-e: 2340-2776.N№ DOI: http://dx.doi.org/10.12795/rea.2015.i32.06

REA 32 (2015):134-150

http://editorial.us.es/es/revista-de-estudios-andaluces 
Figura 1. Evolución del empleo en las ciudades con más de 20.000 habitantes (2000-2006)

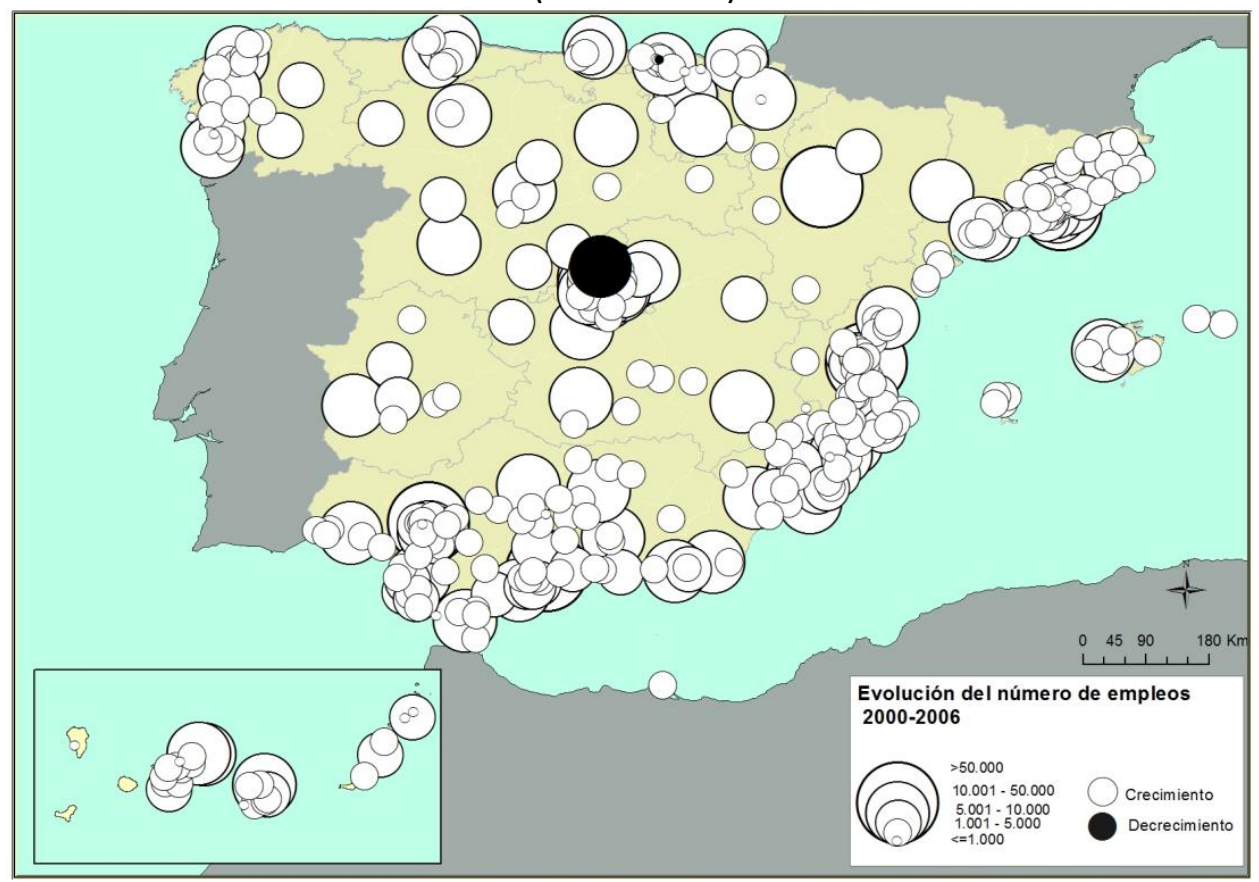

Fuente: Tesorería General de la Seguridad Social y elaboración propia.

De muy distinto signo, sin embargo, es el mapa que muestra los cambios experimentados durante la primera fase de la crisis (2006-2009), siendo ya, por el contrario, numerosos los municipios urbanos que experimentan sustanciales pérdidas de empleo (Figura 2). Dichas pérdidas afectan en mayor medida a las ciudades costeras del Mediterráneo y a las de los dos archipiélagos; junto a las anteriores, el empleo también evolucionaba negativamente en algunas de las ciudades que conforman las principales aglomeraciones metropolitanas. El descontrolado crecimiento experimentado en ellas por el sector de la construcción en los años previos a la crisis explica que el estallido de la burbuja inmobiliaria en estas ciudades resultara especialmente significativo, de tal forma que dicho sector destruyó en su caída a un impresionante número de puestos de trabajo a la vez que generaba importantes impactos territoriales y ambientales (Rodríguez, E. y López, I. 2011; Burriel, E., 2011 y 2014).

Las pérdidas de empleo se generalizan aún más en el periodo comprendido entre 2009 y 2012. En estos años, junto a los efectos de la crisis financiero-inmobiliaria, empiezan a detectarse los producidos por una segunda crisis, derivada en este caso de las políticas de ajuste que, impulsadas por la Unión Europea, empezaron a llevarse a cabo en España (Méndez, R., 2013 a y b; Romero, J., Collado, J.C. y Rodríguez, M.T., 2014). Se trata, pues, de puestos de trabajo perdidos en algunos servicios básicos, como la educación y la sanidad, así como en otras actividades ligadas a la administración del 
Figura 2. Evolución del empleo en las ciudades con más de 20.000 habitantes (2006-2009)

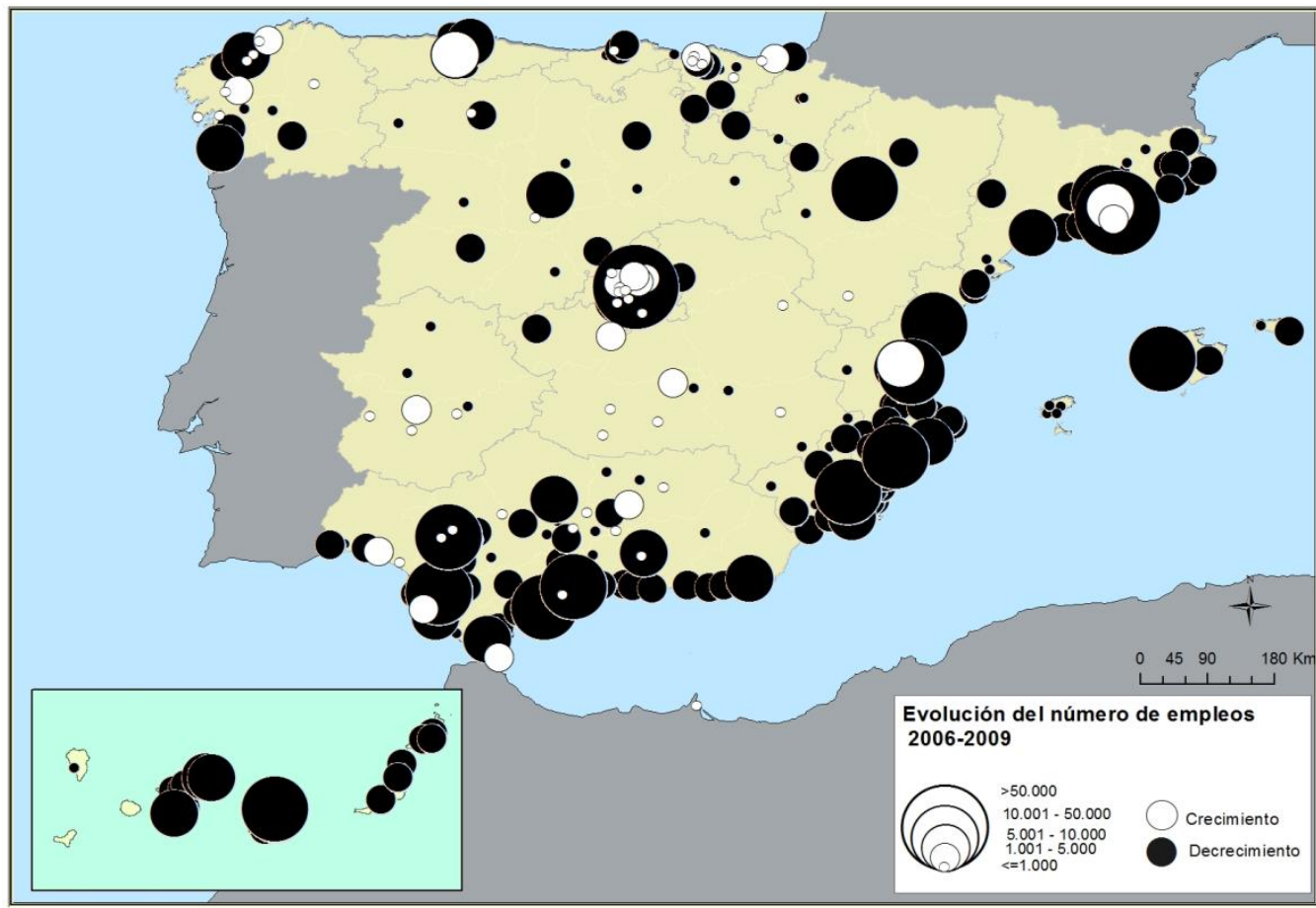

Fuente: Tesorería General de la Seguridad Social y elaboración propia.

Estado, que se localizan sobre todo en las capitales de provincias y en las cabeceras comarcales; ello explica que las pérdidas de empleos afecten a ciudades tanto costeras como del interior, y muchas de ellas de tamaño medio (Figura 3). En este mapa son más numerosos los municipios que pierden empleos, y se distribuyen ya por todo el territorio; no obstante pueden observarse ahora pequeñas excepciones que afectan a municipios costeros e insulares, y muy especialmente a algunos de los integrados en la aglomeración urbana de Madrid.

En definitiva, los mapas anteriores muestran que existen diferencias territoriales en el comportamiento experimentado por el empleo urbano desde que se iniciara la crisis, lo que hay que relacionar con el modelo de crecimiento económico de cada ámbito y con las debilidades o fortalezas de cada uno de ellos (Méndez, 2013 a y b).

\section{DESEQUILIBRIOS URBANOS PROVOCADOS POR LA CRISIS DURANTE LA PRIMERA FASE DE LA CRISIS.}

Se matiza el análisis evolutivo insistiendo aquí en los cambios ocurridos durante la primera fase de la crisis. El cuadro 2, que recoge el ranking de los municipios urbanos considerando la evolución relativa experimentada por el número de afiliaciones a la Seguridad Social entre 2006 y 2009, pone en evidencia los significativos contrastes existentes entre ellas. Las ciudades que mejor han resistido los envites de la crisis conforman distintos grupos. Por una parte, se identifican: ciudades industriales

ISSN: 0212-8594 ISSN-e: 2340-2776.№ DOI: http://dx.doi.org/10.12795/rea.2015.i32.06 
Figura 3. Evolución del empleo en las ciudades con más de 20.000 habitantes

(2009-2012)

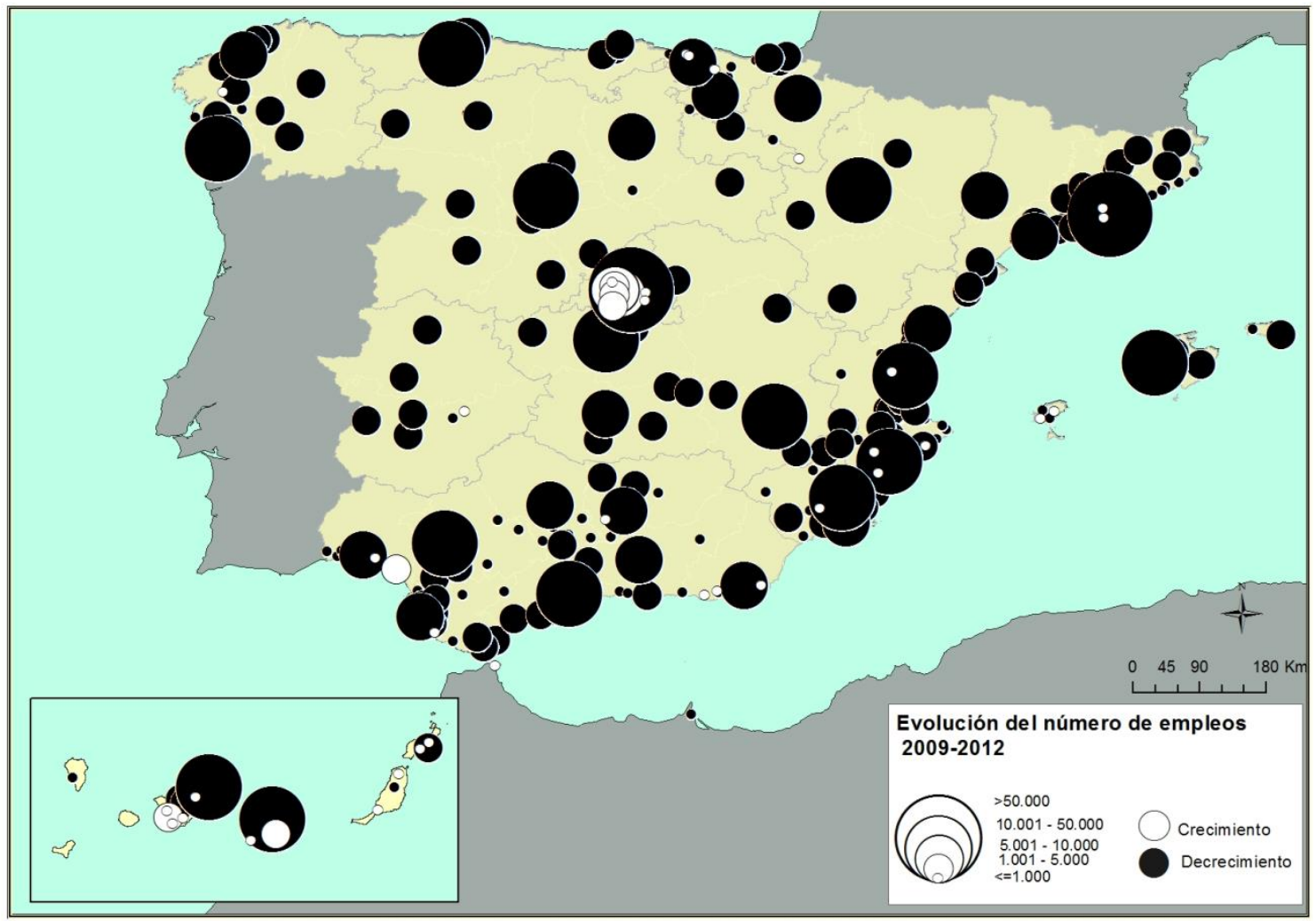

Fuente: Tesorería General de la Seguridad Social y elaboración propia.

tradicionales, mayoritariamente metropolitanas, que han conseguido renovar la estructura de este sector, apostando por actividades de mayor intensidad tecnológica, y diversificando su economía, caso de Paterna (Valencia), Sant Cugat del Vallès (Barcelona) y Erandio (Vizcaya). Por otra, destacan algunas otras ciudades metropolitanas vinculadas a sectores asociados a la economía del conocimiento, donde además se han desarrollado urbanizaciones residenciales de alto nivel, como San Sebastián de los Reyes (Madrid), que han resultado muy atractivas para profesionales de alta cualificación y rentas. Junto a éstas, se incluyen también ciudades con sistemas productivos de doble especialización, asociados tanto al turismo como a la agricultura intensiva; estos son los casos de Moguer y Almonte, en Huelva. A estas ciudades se suman otras con cierto desarrollo industrial y que proveen de servicios a los municipios del entorno, como Narón en La Coruña y Alcázar de San Juan en Ciudad Real. Por su parte, en Ciempozuelos (Madrid), la renovación de la agricultura mediante la incorporación de nuevas técnicas de regadío y el desarrollo de producciones ecológicas ha podido propiciar una mejor resistencia en los inicios de la crisis con el consiguiente mantenimiento de los empleos. En Arroyomolinos, la inauguración en 2003 del centro comercial Madrid-Xanadú y su posterior proceso de ocupación, puede explicar la evolución positiva del empleo. 
Cuadro 2. Ciudades más destacadas según la evolución de las afiliaciones a la Seguridad Social, 2006-2009 (2006=100)

\begin{tabular}{|l|r|l|r|}
\hline \multicolumn{2}{|c|}{$\begin{array}{c}\text { Las } 10 \text { ciudades con valores máximos de } \\
\text { crecimiento }\end{array}$} & $\begin{array}{c}\text { Las 10 ciudades con valores máximos de } \\
\text { decrecimiento }\end{array}$ \\
\hline Moguer (Huelva) & $34,56 \%$ & Quart de Poblet (Valencia) & $-35,73 \%$ \\
\hline Alcázar de San Juan (Toledo) & $20,71 \%$ & Chiclana de la Frontera (Cádiz) & $-28,61 \%$ \\
\hline Arroyomolinos (Madrid) & $20,12 \%$ & Pinto (Madrid) & $-27,99 \%$ \\
\hline Paterna (Valencia) & $16,79 \%$ & Rojales (Alicante) & $-25,28 \%$ \\
\hline Sant Cugat del Vallès (Barcelona) & $13,15 \%$ & Estepona (Málaga) & $-24,83 \%$ \\
\hline Narón (La Coruña) & $11,61 \%$ & Alhaurín el Grande (Málaga) & $-24,60 \%$ \\
\hline San Sebastián de los Reyes (Madrid) & $10,57 \%$ & Cártama (Málaga) & $-24,31 \%$ \\
\hline Ciempozuelos (Madrid) & $9,51 \%$ & Coín (Málaga) & $-24,28 \%$ \\
\hline Almonte (Huelva) & $9,37 \%$ & Arcos de la Frontera (Cádiz) & $-24,14 \%$ \\
\hline Erandio (Vizcaya) & $8,84 \%$ & Ayamonte (Huelva) & $-9,89 \%$ \\
\hline
\end{tabular}

Fuente: Tesorería General de la Seguridad Social y elaboración propia.

A diferencia de ello, el mayor descenso ha tenido lugar en ciudades con menos de 50.000 habitantes, situadas en el litoral y su traspaís con un alto grado de dependencia de la construcción y el turismo, sectores caracterizados por la temporalidad y baja cualificación de los empleos (Estepona, Alhaurín el Grande, Coín, y Cártama en Málaga, Ayamonte en Huelva, Chiclana en Cádiz y Rojales en Alicante). Estos municipios, además de ser mayoritariamente andaluces, forman parte, sobre todo, de zonas turísticas de expansión reciente surgidas como consecuencia de la colmatación y saturación de ámbitos más tradicionales y que, por tanto, resultaban sumamente atractivos para la voraz actividad inmobiliaria de las últimas décadas. Otro grupo de ciudades lo conforman Quart de Poblet (Valencia) y Pinto (Madrid); éstas se caracterizan por su localización en las periferias metropolitanas y por una mayor presencia industrial, aunque con ciertas diferencias entre las mismas. Así, Pinto cuenta con un sector industrial más diversificado, junto con un importante desarrollo de las actividades de distribución, propias de un municipio enclavado en la periferia metropolitana. A diferencia de ello, Quart de Poblet tiene un sector industrial con una mayor especialización, concretamente, en el sector cerámico, cuya demanda está directamente relacionada con la construcción. Por su parte, Arcos de la Frontera (Cádiz), se corresponde con una agrociudad cuya estructura económica está aún vinculada a la explotación y transformación de recursos agrarios, donde el turismo interior no ha logrado dinamizar la economía; la central térmica de ciclo combinado que se instaló en el municipio el año 2005, generó un fuerte aumento del PIB, pero sin crear apenas empleo.

\section{DESEQUILIBRIOS URBANOS PROVOCADOS POR LAS POLÍTICAS DE AUSTERIDAD.}

Para completar el apartado anterior, el cuadro 3 recoge las 10 ciudades que más destacan positiva y negativamente según la evolución de las afiliaciones en la segunda etapa de la crisis, 2009 y 2012. Si en un primer momento, la desaceleración económica

ISSN: 0212-8594 ISSN-e: 2340-2776.N№ DOI: http://dx.doi.org/10.12795/rea.2015.i32.06

REA 32 (2015):134-150

http://editorial.us.es/es/revista-de-estudios-andaluces 
Cuadro 3. Ciudades más destacadas según la evolución de las afiliaciones a la Seguridad Social, 2009-2012 (2009=100)

\begin{tabular}{|l|r|l|r|}
\hline \multicolumn{2}{|c|}{$\begin{array}{c}\text { Las } 10 \text { ciudades con valores máximos de } \\
\text { crecimiento }\end{array}$} & \multicolumn{2}{c|}{$\begin{array}{c}\text { Las 10 ciudades con valores máximos de } \\
\text { decrecimiento }\end{array}$} \\
\hline Majadahonda (Madrid) & $24,59 \%$ & La Orotava (Santa Cruz de Tenerife) & $-26,16 \%$ \\
\hline Arroyomolinos (Madrid) & $23,79 \%$ & Cullera (Valencia) & $-22,61 \%$ \\
\hline Ingenio (Las Palmas) & $23,71 \%$ & Tomelloso (Ciudad Real) & $-21,17 \%$ \\
\hline Boadilla del Monte (Madrid) & $18,80 \%$ & Lalín (Pontevedra) & $-21,12 \%$ \\
\hline Almonte (Huelva) & $13,27 \%$ & Langreo (Asturias) & $-20,97 \%$ \\
\hline Ames (La Coruña) & $13,21 \%$ & Xirivella (Valencia) & $-19,75 \%$ \\
\hline Adeje (Santa Cruz de Tenerife) & $12,49 \%$ & Sant Vicenç dels Horts (Barcelona) & $-19,64 \%$ \\
\hline La Oliva (Las Palmas) & $11,31 \%$ & Hellín (Albacete) & $-19,45 \%$ \\
\hline Pozuelo de Alarcón (Madrid) & $10,34 \%$ & Coslada (Madrid) & $-19,44 \%$ \\
\hline Mogán (Las Palmas) & $9,90 \%$ & Alzira (Valencia) & $-18,47 \%$ \\
\hline
\end{tabular}

Fuente: Tesorería General de la Seguridad Social y elaboración propia.

afectó con mayor intensidad a los municipios urbanos más vinculados al desarrollo inmobiliario de los años previos a la crisis; en una segunda, la reducción de las rentas y el consumo, como consecuencia de la pérdida de empleos y de la aplicación de políticas de austeridad, explican que se extiendan sus efectos por el territorio y que, prácticamente, cualquier tipo de ciudad se vea afectada, en mayor o menor medida, por la crisis. Aquellas que mejor resisten son las que acogen a segmentos poblacionales con mayores rentas (Majadahonda, Boadilla del Monte y Pozuelo de Alarcón en Madrid), las vinculadas a un turismo internacional (Ingenio, La Oliva y Mogán en Las Palmas; Adeje en Santa Cruz de Tenerife) y aquellas con sistemas productivos con doble especialización, asociados al turismo y a la agricultura intensiva (Almonte en Huelva).

En la segunda etapa de la crisis, es difícil establecer cierta similitud entre las ciudades más afectadas, pues entre las mismas se observan diferencias significativas. Así, se pueden encontrar ciudades especializadas en el turismo, tanto internacional como nacional (La Orotava en Santa Cruz de Tenerife y Cullera en Valencia), las que cuentan con una industria suministradora de bienes para la construcción (Xirivella en Valencia y Hellín en Albacete) o de productos de consumo final como el textil (Lalín en Pontevedra), y las que tienen economías más diversificadas, ya sea porque se sitúan en áreas metropolitanas o porque ejercen de cabeceras comarcales (Tomelloso en Ciudad Real, Langreo en Asturias, Sant Vicenç dels Horts en Barcelona, y Coslada en Madrid).

\section{HACIA UNATIPOLOGÍA DE CIUDADES, SEGÚN LA EVOLUCIÓN DEL EMPLEO Y LA TASA DE ACTIVIDAD.}

El análisis sobre los efectos territoriales de la crisis en el empleo se completa finalmente realizando una tipología que vincula la evolución del número de afiliaciones a la Seguridad Social entre 2006 y 2012 con la tasa de actividad en este último año, 
considerando como tal la relación entre las afiliaciones a la Seguridad Social y la población en edad activa (\%).

Fruto de esta asociación se identifican cuatro situaciones: Las ciudades correspondientes al tipo A, caracterizadas por haber experimentado un crecimiento en el número de afiliaciones más positivo que la media nacional y contar con una relación entre éstas y la población en edad activa superior al $50 \%$. Forman parte del tipo B las que han conocido una evolución de las afiliaciones más positiva que la del conjunto, aunque cuentan con un porcentaje de afiliaciones en relación a la población en edad activa inferior al $50 \%$. Se incluyen en el tipo $\mathrm{C}$ aquellas que pese a haber conocido una evolución del número de afiliaciones más negativa que la de la media nacional, alcanzan en 2012 cifras superiores al 50 \% de afiliados respecto a la población en edad activa. Conforman, por último, el tipo $\mathrm{D}$ las que en las cifras correspondientes a los dos indicadores utilizados se sitúan por debajo de ambos parámetros (cuadro 4).

Cuadro 4. Tipología de ciudades según los impactos de la crisis sobre el empleo.

\begin{tabular}{|l|c|c|c|}
\hline TIPO & $\begin{array}{c}\text { Evolución de las } \\
\text { afiliaciones respecto a la } \\
\text { media, 2006-2012 }\end{array}$ & $\begin{array}{c}\text { Afiliaciones/Población edad } \\
\text { activa, 2012 (>50\%) }\end{array}$ & No Ciudades \\
\hline A & + & + & $18(4,51 \%)$ \\
\hline B & + & - & $14(3,51 \%)$ \\
\hline C & - & + & $104(26,07 \%)$ \\
\hline D & - & - & $263(65,91 \%)$ \\
\hline TOTAL & & & $399(100 \%)$ \\
\hline
\end{tabular}

Fuente: Tesorería General de la Seguridad Social y elaboración propia

Tal y como puede observarse en el cuadro 4, la mayoría de los municipios urbanos españoles $(65,91 \%)$ han experimentado una reducción del número de las afiliaciones que ha conducido a unas tasas de actividad inferiores al 50\%, derivándose de ello una alta tasa de población dependiente y, por tanto, vulnerabilidad y fragilidad social (tipo D). En este grupo se incluyen desde ciudades industriales metropolitanas que ya habían conocido un duro proceso de reestructuración y cierre de empresas previo a la crisis (Alcalá de Henares, Alcalá de Guadaíra, San Fernando, Mieres, Portugalete, Rentería, Sestao...), hasta ciudades con distritos industriales vinculados a la construcción (Lucena, Alcoy, Crevillente, Burriana, La Vall d'Uixó...), pero también a otros productos como el textil (Lalín, Alcoy...). Junto a ellas, se identifican ciudades metropolitanas con una clara funcionalidad residencial, que han acogido a los sectores sociales de rentas más bajas (Parla, Móstoles, Santa Coloma de Gramenet...) y ciudades, mayoritariamente mediterráneas, dependientes del turismo y la actividad constructiva asociada al mismo (Calviá, Rojales, Rincón de la Victoria, Santa Pola, Torrevieja...). 
En el extremo opuesto (tipo A), se encuentran aquellos municipios urbanos que no sólo han visto evolucionar el empleo mejor que la media sino que cuentan con unas tasas de actividad superiores al $50 \%$. Responden a este tipo dieciocho ciudades que sólo representan un 4,51 \% del total; entre ellas pueden identificarse ciudades del área metropolitana de Madrid que acogen a los profesionales de alta cualificación y sectores sociales de mayores rentas (Majadahonda, San Sebastián de los Reyes, Boadilla del Monte, Tres cantos, Pozuelo de Alarcón, Alcobendas y Las Rozas). Se incluyen también en este tipo ciudades industriales que han conseguido renovar la estructura de este sector, apostando por actividades con mayores insumos de conocimiento $y$ diversificando su economía (Paterna, El Prat de Llobregat, Sant Cugat del Vallès, Erandio, Leioa y Mondragón); así como algunas ligadas al turismo internacional (Adeje y Mogán), o que cuentan con sistemas productivos locales asociados conjuntamente a la agricultura intensiva y el turismo (Níjar, Almonte y Moguer).

El número de ciudades que se reparte entre los dos tipos intermedios, B y $\mathrm{C}$, es muy desigual, pues mientras catorce se comportan según lo establecido en el tipo $B(3,51 \%$ del total) son ciento cuatro las que lo hacen según el $C(26,07 \%)$. Respecto a las primeras, coinciden con ciudades medias cabeceras comarcales (Alcázar de San Juan, Cabra y Don Benito), con ciudades metropolitanas con una expansión urbana más reciente que ha sido ocupada por clases medias (Arroyomolinos, Rivas-Vaciamadrid, Ames y Armilla), ciudades vinculadas al turismo con un porcentaje elevado de extranjeros (Sant Josep de Sa Talaia y Santa Eulalia del Río), y ciudades con un peso significativo de la industria agroalimentaria (Piélagos y Ribeira); a ellas se suman las dos ciudades autónomas (Melilla y Ceuta). Por último, entre las ciudades incluidas en el tipo $C$, se encuentran las mayores ciudades, lo que pone en evidencia que la crisis es un fenómeno con una clara vocación urbana, pero que la propia complejidad y diversidad de este tipo de espacios motiva que una destrucción de empleos significativa siga siendo compatible con unas tasas de actividad altas.

\section{ALGUNAS CONSIDERACIONES FINALES.}

Son muchos los efectos adversos que la crisis está provocando y entre los más destacados se encuentra la reducción experimentada por el empleo. Este hecho está directamente relacionado, por una parte, con el proceso de reestructuración del desregulado e hipertrofiado sector financiero y, por otra, con el estallido de la llamada burbuja inmobiliaria. Pero no puede olvidarse que se añaden a los anteriores los impactos provocados por la aplicación de políticas neoliberales de desregulación y privatización económica que están incidiendo más intensamente en los sectores sociales de más baja renta contribuyendo así a reducir la cohesión social.

Unos y otros procesos ponen además en evidencia que la crisis está afectando al crecimiento de las desigualdades no sólo sociales sino también territoriales, contribuyendo a generar nuevos desequilibrios y contradicciones que se hacen más

ISSN: 0212-8594 ISSN-e: 2340-2776.№ DOI: http://dx.doi.org/10.12795/rea.2015.i32.06

REA 32 (2015):134-150

http://editorial.us.es/es/revista-de-estudios-andaluces 
patentes a escala local. En este contexto, los espacios urbanos resultan de interés para analizar estos impactos.

España es uno de los países más afectados por la crisis y, muy especialmente, su mercado laboral. Si la explosión de la burbuja inmobiliaria destruyó un considerable número de empleos, las políticas de austeridad que pretenden controlar la crisis no han hecho sino potenciar aún más tales pérdidas. La mayor incidencia de uno y otro proceso ha permitido diferenciar dos etapas en la evolución de la crisis: una primera asociada al comportamiento de los sectores inmobiliario y financiero; $y$, una segunda, relacionada con la reducción del gasto público en los servicios.

En cuanto a los efectos generados en las ciudades españolas por dichos procesos, pueden establecerse diferencias según su volumen de población:

- Se mantiene el desequilibrio económico entre los municipios, puesto que sólo en los que superan los 100.000 habitantes el peso relativo de las afiliaciones a la Seguridad Social supera al de la población.

- Durante los primeros años de la crisis, la pérdida de empleos es más significativa en los municipios urbanos de mayor y menor tamaño.

- En la segunda etapa, el descenso del número de afiliaciones a la Seguridad Social se intensifica en los municipios entre 50.000 y 500.000 habitantes, mientras se frena tanto en las ciudades mayores como en las menores.

- La relación entre el número de afiliaciones y la población en edad activa es más alta en los municipios con más de 100.000 habitantes.

Aunque las transformaciones territoriales son lentas y, por tanto, no fáciles de apreciar, se pueden observar ya ciertas tendencias territoriales que requieren atención:

- Ya en la primera fase de la crisis son numerosas las ciudades que pierden empleos, destacando las situadas en la franja costera mediterránea, en los dos archipiélagos y en las principales aglomeraciones metropolitanas; lo que pone claramente en evidencia la responsabilidad y el protagonismo que el sector inmobiliario ha tenido en la destrucción de empleo.

- Los efectos de las políticas de austeridad se observan posteriormente difundiéndose la crisis por todo el territorio. En esta segunda fase se ven también afectadas las capitales de provinciales y las cabeceras comarcales, que son las que fundamentalmente concentran los servicios, mientras se detectan, sin embargo, cierto crecimiento del empleo en determinados municipios costeros e insulares, y en algunos de los que conforman la aglomeración urbana de Madrid.

- Para profundizar en el análisis, se establece una tipología que permite matizar las conclusiones anteriores. Se pone con ella de manifiesto que tanto la localización como la especialización funcional de las distintas ciudades parecen ser factores explicativos del mayor o menor grado de afección de la crisis durante sus dos fases. 
En definitiva, el análisis de la incidencia de la crisis en el empleo de las ciudades españolas mayores de 20.000 habitantes pone de manifiesto que ésta está siendo muy desigual, y que las diferencias existentes entre ellas deben ser tenidas en cuenta para diseñar políticas adaptadas a las distintas realidades que puedan activar nuevos procesos de desarrollo territorial.

Por último hay que tener en cuenta que, aunque del análisis hasta ahora realizado sobre la incidencia territorial de la crisis han podido ya sacarse algunas conclusiones, no puede dejarse al margen el hecho de que no se trata más que de una primera aproximación que deberá ser completada con trabajos posteriores. No hay que olvidar que profundizar en esta temática exige considerar no sólo la evolución del número de afiliaciones a la Seguridad Social, sino también en qué sectores de actividad se insertan éstas, cuántos nuevos contratos se están firmando y de qué tipos, cómo evoluciona el número de parados, a qué grupos sociales les afecta... Sólo así será posible concluir si la crisis puede estar modificando y en qué medida el modelo territorial español, aportando, además, información que permita diseñar políticas públicas que se adapten a las distintas realidades locales.

\section{BIBLIOGRAFÍA}

Albertos, J.M. y Sánchez, J.L. coords. (2014): Geografía de la crisis económica en España, Valencia, Universidad de Valencia.

Álvarez Peralta, I.; Luengo Escalonilla, F. y Uxó González, J. (2013): Fracturas y crisis en Europa, Eudeba y Clave Intelectual, Buenos Aires-Madrid.

Ayala, I.; Martínez, R. y Ruiz Huerta, J. (2013): “Desigualdad y redistribución en los países de la OCDE". En Primer informe sobre la desigualdad en España. Fundación Alternativas, Madrid.

Askenazy, P. y Méhaut, P. (2012): “Dotar de un nuevo porvenir al trabajo” En VV.AA. Los economistas aterrados, Cambiar de economía. Catarata, Madrid, pp. 190-204.

Boccara, F.; Le Héron, E. y Plihon, D. (2012): “Por un sistema financiero emancipado de los mercados financieros" en VV.AA. Los economistas aterrados, Cambiar de economía. Catarata, Madrid, pp. 230-250.

Burriel, E. (2011): "Subversion of land-use plans and the housing bubble in Spain" Urban Research \& Practice, Vol. 4, no 3, pp. 232-249.

Burriel, E. (2011): "Subversion of land-use plans and the housing bubble in Spain" Urban Research \& Practice, Vol. 4, no 3, pp. 232-249.

ISSN: 0212-8594 ISSN-e: 2340-2776.№ DOI: http://dx.doi.org/10.12795/rea.2015.i32.06 
http://dx.doi.org/10.1080/17535069.2011.616743

Castaño, C. (1994): Tecnología, empleo y trabajo en España, Alianza, Madrid.

Corpataux, J.; Crevoisier, O. and Theurillat, T. (2009): "The expansion of the finance industry and its impacto in the economy: a territorial 148 Approach base on Swiss pension funds", Economic Geography, no 85 (3), pp. 313-334.

Daher, A. (2013): "El sector inmobiliario y las crisis económicas" EURE. Revista Latinoamericana de Estudios Urbano- Regionales, Vol 39, no 118, pp. 47-76.

http://dx.doi.org/10.4067/S0250-71612013000300003

Dévoluy, M. ET AL (2012): "La política económica en Europa” en VVAA Los Economistas aterrados, Cambiar de Economía, Madrid, Catarata, pp. 49-74.

Ekaizer, E. (2012): Indecentes. Espasa, Madrid.

Etxzarreta, M., Coord. (1995): La reestructuración del capitalismo en España 1970-1990, Icaria-Fuhem, Barcelona.

Fernández Durán, R. (2011): La quiebra del capitalismo global: 2000-2030. Virus Editorial, Bilbao.

George, S. (2010): Sus crisis, nuestras soluciones. Barcelona Icaria-Intermón-Oxfam.

Harvey, D. (2012 a): El enigma del capital y las crisis del capitalismo. Akal, Madrid.

Harvey, D. (2012 b): "Las raíces urbanas de las crisis financieras" En Bell, Borja y Corti Eds. Ciudades, una ecuación imposible, Icaria, Barcelona, pp. 321-358.

Intermón Oxfam (2012): Crisis, desigualdad y pobreza. Aprendizaje desde el mundo en desarrollo ante los recortes sociales en España,

<http://www.oxfamintermon.org/es/informate/publicaciones/estudios > [26 de julio de 2014]

López, I. y Rodríguez, E. (2010): Fin de ciclo. Financiarización. Territorio y sociedad de propietarios en la onda larga del capitalismo hispano (1959-2010), Traficantes de Sueños, Madrid.

Lordon, F. (2012): "La pavorosa pasividad de la re-regulación financiera" en Los economistas aterrados, Cambiar de economía, Catarata, Madrid, pp.251-281.

ISSN: 0212-8594 ISSN-e: 2340-2776.N№ DOI: http://dx.doi.org/10.12795/rea.2015.i32.06 
Martin, R. (2011): "The local geographies of the financial crisis: from the housing bubble to economic recession and beyond" Journal of Economic Geography, vol. 11 (4), pp. 587618.

Martin, R. (2012): "Regional economic resilience, hysteresis and recessionary shocks" Journal of Economic Geography, vol.12, pp.1-32. http://dx.doi.org/10.1093/jeg/lbr019

Méndez, R. (1998): "Hacia una nueva división espacial del trabajo en España" en De Mattos-Hiernaux-Restrepo Comp. Globalización y Territorio. Impactos y perspectivas, Pontificia Universidad Católica de Chile. Instituto de Estudios Urbanos y Fondo de Cultura Económica, Santiago de Chile, pp. 120-152.

Méndez, R. (2013 a): Las escalas de la crisis. Ciudades y desempleo en España, Fundación 1ㅇ de Mayo, Colección Estudios <www.1mayo.cooo.es> [22 de julio de 2014]

Méndez, R. (2013 b): “Crisis económica, vulnerabilidad urbana y desempleo en España" en Ciudad y Territorio Estudios Territoriales, XLV (178), pp.1-19

Méndez, R. (2014): "Crisis económica y reconfiguraciones territoriales" en AlbertosSánchez coords. Geografía de la crisis económica en España, Universidad de Valencia, Valencia, pp. 17-38.

Morin, E. (2011): La Vía. Para el futuro de la humanidad, Paidós Estado y Sociedad, Barcelona.

Naredo, J.M. (2009): "La cara oculta de la crisis. El fin del boom inmobiliario y sus consecuencias" Revista de Economía Crítica № 7, pp. 313-340.

Observatorio Metropolitano (2011): La crisis que viene. Algunas notas para afrontar esta década, Traficantes de sueños, Madrid.

Oxfam Intermon (2013): La trampa de la austeridad. El verdadero coste de la desigualdad en Europa.

<http://www.oxfamintermon.org/es/informate/publicaciones/estudios> [21 de julio de 2014]

Rocha, F. y Aragón, J. (2012): "La crisis económica y sus efectos sobre el empleo" Colección Informes, № 55, Fundación 1ㅇ de Mayo.

Rodríguez, E. y López, I. (2011): “Del auge al colapso. El modelo financiero-inmobiliario de la economía española (1995-2010)" Economía Crítica, № 12, pp. 39-64.

Romero, J. (2010): "Construcción residencial y gobierno del territorio en España. De la burbuja especulativa a la recesión. Causas y consecuencias" Cuadernos Geográficos, № 47, pp. 17-46.

ISSN: 0212-8594 ISSN-e: 2340-2776.N№ DOI: http://dx.doi.org/10.12795/rea.2015.i32.06 
Romero, J.; Collado, J.C. y Rodríguez, M.T. (2014): “La economía política de la desigualdad. Los recortes en el sector público y sus repercusiones para el Estado de Bienestar en España" en Albertos Puebla, J.M.-Sánchez Hernández, J.L. Coords. (2014): Geografía de la crisis económica en España, Publicaciones de la Universidad de Valencia, Valencia, pp. 329-376.

Sánchez Hernández, J.L. (2013): "Sensibilidad y resiliencia de las regiones españolas durante las crisis económicas (1976-2011)" en Llusa \&Feliu \&Paunero. Edits, Crisis económica e impactos territoriales, V Jornadas de Geografía Económica, 2012, GGE (AGE), Girona, Dpto. de Geografía de la Universidad de Girona, pp.74-96.

Sterdyniak, H. (2012): “¿Que política económica? Muertes y resurrecciones del keynesianismo" en Los economistas aterrados, Cambiar de economía, Catarata, Madrid, pp. 21-48.

Toharia, L. (1992): "El mercado de trabajo español. Algunos datos y estudios", Revista de Estudios Regionales, Na 31, pp. 105-114.

Torres López, J. (2011): Contra la crisis, otra economía y otro modo de vivir. Ed. HOAC, Móstoles (Madrid).

Touraine, A. (2011): Después de la crisis, Paidos, Barcelona.

VVAA (2012): Los economistas aterrados. Cambiar de economía, Catarata, Madrid. 